\title{
Breast Contour Detection with Stable Paths
}

\author{
Jaime S. Cardoso, Ricardo Sousa, Luís F. Teixeira, and M.J. Cardoso \\ INESC Porto, Faculdade de Engenharia, Universidade do Porto, Portugal \\ \{jaime.cardoso,rsousa, luis.f.teixeira\}@inescporto.pt, \\ mjcard@med.up.pt
}

\begin{abstract}
Breast cancer conservative treatment (BCCT), due to its proven oncological safety, is considered, when feasible, the gold standard of breast cancer treatment. However, aesthetic results are heterogeneous and difficult to evaluate in a standardized way, due to the lack of reproducibility of the subjective methods usually applied. The objective assessment methods, considered in the past as being less capable of evaluating all aspects of BCCT, are nowadays being preferred to overcome the drawbacks of the subjective evaluation. A computer-aided medical system was recently developed to objectively and automatically evaluate the aesthetic result of BCCT. In this system, the detection of the breast contour on the patient's digital photograph is a necessary step to extract the features subsequently used in the evaluation process. In this paper an algorithm based on the shortest path on a graph is proposed to detect automatically the breast contour. The proposed method extends an existing semi-automatic algorithm for the same purpose. A comprehensive comparison with manually-drawn contours reveals the strength of the proposed method.
\end{abstract}

\section{Introduction}

Breast cancer is the most common cancer to affect women in Europe and as 10-year survival from the disease now exceeds $80 \%$, many women are expected to live a long time with the aesthetic consequences of their treatment. The importance of good aesthetic outcome is well recognised by experts in this field although it is well known that this is often not achieved. In breast-conserving surgery for example, approximately $30 \%$ of women will have a suboptimal or poor aesthetic outcome.

A significant obstacle in auditing this problem and evaluating techniques for improving it has been the absence of a standard method for measuring aesthetic outcome. Most commonly used methods involve subjective assessment by an expert panel. All these methods are subject to significant intra-observer and inter-observer variability. So, there is a need to replace or enhance human expert evaluation of the aesthetic results of breast surgery with a validated objective tool. This needs to be easy to employ, completely reproducible and acceptable to those who would be evaluated.

Initially, objective methods consisted of the comparison between the two breasts with simple measurements marked directly in patients or in photographs [1,2]. The correlation of objective measurements with subjective overall evaluation has been reported by several authors [2]. Nevertheless, objective methods usually assessed just one aspect of cosmesis such as nipple symmetry. Trying to overcome the sense that objective asymmetry measurements were insufficient, other groups proposed the sum of the individual

A. Fred, J. Filipe, and H. Gamboa (Eds.): BIOSTEC 2008, CCIS 25, pp. 439-452, 2008.

(c) Springer-Verlag Berlin Heidelberg 2008 


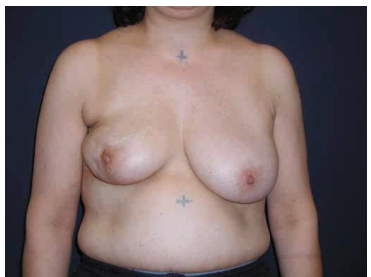

(a)

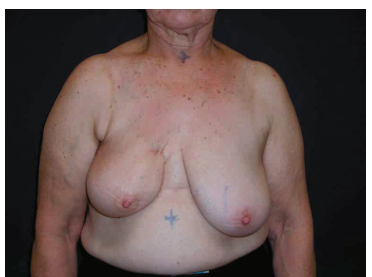

(b)

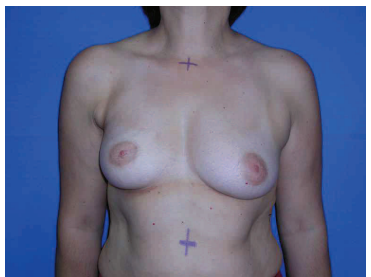

(c)

Fig. 1. Typical photographs

scores of subjective and objective individual indices [3]. More recently, a computeraided medical system was developed to objectively and automatically perform the aesthetic evaluation of BCCT [4]. The development of this system entailed the automatic extraction of several features from the patient's photographs (Fig. 1), capturing some of the factors considered to have impact on the overall cosmetic result: breast asymmetry, skin colour changes due to the radiotherapy treatment and surgical scar appearance. In a second phase, a support vector machine classifier was trained to predict the overall cosmetic result from the recorded features [4].

In order to extract the identified relevant features from the image, the detection of the breast contour is necessary. In $[5,6]$ we describe a semi-automatic method for the detection of the breast contour. The user has to manually identify the two endpoints of the breast contour. Subsequently, the algorithm automatically finds the contour in-between. The algorithm has been implemented in the system described above: the software automatically finds the contours, extracts relevant features and outputs a predicted overall cosmetic assessment (excellent, good, fair, or poor).

Here, we improve on the work of [5,6] in two different directions. First, we present an algorithm for the automatic detection of the endpoints of the breast contour, thus eliminating any user input from the process. Therefore a totally automatic breast contour detection is achieved. Next, we provide a thorough evaluation of the performance of the proposed method against manually-drawn breast contours. Standard metrics are employed to compare two contours.

Before presenting the proposed approach, and for completeness, we recover the framework for breast contour detection between two known endpoints of [5,6]. Then, in Section 3 we detail how to automatically find the endpoints of a breast contour. Examples are provided and a performance analysis is conducted in Section 4. Finally, in Section 5, we conclude the paper and present possible directions of future work.

\section{A Shortest Path Approach to Contour Detection}

When the two endpoints of the breast contour are known, we are left with the problem of finding the path between both endpoints going through the breast contour. Since the interior of the breast itself is essentially free of edges, the path we are looking for is the shortest path between the two endpoints, if paths (almost) entirely through edge pixels are favoured. More formally, let $s$ and $t$ be two pixels of the image and $\mathcal{P}_{s, t}$ a path over 
the image connecting them. We are interested in finding the path $\mathcal{P}$ that optimizes some predefined distance $d(s, t)$. This criterion should embed the need to favour edge pixels.

In the work to be detailed, the image grid is considered as a graph with pixels as nodes and edges connecting neighbouring pixels. Therefore, some graph concepts are in order.

\subsection{Background Knowledge}

A graph $G=(V, A)$ is composed of two sets $V$ and $A$. $V$ is the set of nodes, and $A$ the set of arcs $(p, q), p, q \in V$. The graph is weighted if a weight $w(p, q)$ is associated to each arc. The weight of each arc, $w(p, q)$, is a function of pixels values and pixels relative positions. A path from vertex (pixel) $v_{1}$ to vertex (pixel) $v_{n}$ is a list of unique vertices $v_{1}, v_{2}, \ldots, v_{n}$, with $v_{i-1}$ and $v_{i}$ corresponding to neighbour pixels. The total cost of a path is the sum of each arc weight in the path $\sum_{i=2}^{n} w\left(v_{i-1}, v_{i}\right)$.

A path from a source vertex $v$ to a target vertex $u$ is said to be the shortest path if its total cost is minimum among all $v$-to- $u$ paths. The distance between a source vertex $v$ and a target vertex $u$ on a graph, $d(v, u)$, is the total cost of the shortest path between $v$ and $u$.

A path from a source vertex $v$ to a sub-graph $\Omega$ is said to be the shortest path between $v$ and $\Omega$ if its total cost is minimum among all $v$-to- $u \in \Omega$ paths. The distance from a node $v$ to a sub-graph $\Omega, d(v, \Omega)$, is the total cost of the shortest path between $v$ and $\Omega$ :

$$
d(v, \Omega)=\min _{u \in \Omega} d(v, u) .
$$

A path from a sub-graph $\Omega_{1}$ to a sub-graph $\Omega_{2}$ is said to be the shortest path between $\Omega_{1}$ and $\Omega_{2}$ if its total cost is minimum among all $v \in \Omega_{1}$-to- $u \in \Omega_{2}$ paths. The distance from a sub-graph $\Omega_{1}$ to a sub-graph $\Omega_{2}, d\left(\Omega_{1}, \Omega_{2}\right)$, is the total cost of the shortest path between $\Omega_{1}$ and $\Omega_{2}$ :

$$
d\left(\Omega_{1}, \Omega_{2}\right)=\min _{v \in \Omega_{1}, u \in \Omega_{2}} d(v, u) .
$$

In graph theory, the shortest-path problem seeks the shortest path connecting two nodes; efficient algorithms are available to solve this problem, such as the well-known Dijkstra algorithm [7].

\subsection{Base Algorithm for Breast Contour Detection}

If the weight assigned to an arc in the graph captures the edge strength of the incident pixels, finding the best contour translates into computing the minimum accumulated weight along all possible curves connecting $s$ and $t$ :

$$
d(s, t)=\min _{\mathcal{P}_{s, t}} \sum w(p, q) .
$$

Note that, if we ignore the weight component, we are simply computing the regular Euclidian distance between $s$ and $t$ along the path $\mathcal{P}_{s, t}$ (which will be a straight line for the shortest path). Therefore, to detect the breast contour the framework in [5] encompasses: 
1. A gradient computation of the original image. In a broader view, this can be replaced by any feature extraction process that emphasizes the pixels we are seeking for.

2. Consider the gradient image as a weighted graph with pixels as nodes and arcs connecting neighbouring pixels. Assign to an arc an weight $w$ determined by the gradient values of the two incident pixels.

The weight of the arc connecting 4-neighbour pixels $p$ and $q$ was expressed as an exponential law

$$
\widehat{f}(g)=\alpha \exp (\beta(255-g))+\gamma,
$$

with $\alpha, \beta, \gamma \in \mathbb{R}$ and $g$ is the minimum of the gradient computed on the two incident pixels. For 8 -neighbour pixels the weight was set to $\sqrt{2}$ times that value. The parameters $\alpha, \beta$ and $\gamma$ were experimentally tuned using a grid search method, yielding $\alpha=0.15, \beta=0.0208$ and $\gamma=1.85$.

\subsection{Breast Contour Detection with Shape Priors}

To enforce shape constraints, deformable templates are often used. Prototype-based deformable templates are usually constructed from a set of training examples. Then principal component analysis is applied to define an average template shape and modes of variation $[8,9,10]$. Unfortunately, when graph algorithms are used, the cost function is influenced by only two consecutive pixels at a time, whereas more pixels would be needed to encode shape information. Nevertheless, graph algorithms have the advantage over gradient descent-typical on active contours - in that they recover the optimal solution and do not get trapped in local minima. Therefore, in [6], we proposed different approaches to encode the a priori shape knowledge in the graph design. The weight function was modified by a term reflecting our knowledge of the shape probability. The shortest path problem is then formulated on this modified weighted graph. The key advantage of this approach is that the shortest path computation is left unchanged and hence the procedure remains efficient and discovers the optimal solution.

Although parametric models may seem appealing, they did not achieve a performance as good as non-parametric models for this specific task [6]. Therefore, we only summarize here the latter.

Non-parametric Mask Prior. Supposing that we have a training set $C=\left\{C_{i} \mid i=\right.$ $1,2, \ldots, M\}$ of $M$ registered breast contours, manually outlined, we propose to construct a nonparametric breast shape prior by defining an admissible breast contour region. Considering the breast contours as closed curves (by connecting the start and ending points), we defined the difference between the reunion and the intersection of all breast contour regions as

$$
\mathcal{R}=\cup_{i=1}^{M} \mathcal{C}_{i}-\cap_{i=1}^{M} \mathcal{C}_{i} .
$$

The region $\mathcal{R}$ is the smallest simply connected region that contains all breast contours on the training set. If the training set is representative of the population-it was drawn from the underlying breast shape and size distribution-, one expects that unseen breast contours will be found (almost) entirely inside the region $\mathcal{R}$. 
To incorporate this prior information into the breast contour detection process, the image is represented by its Euclidean distance map $\Phi\left(x_{i}, y_{i}\right)$, where the distance from each pixel is computed to the nearest pixel belonging to the region $\mathcal{R}$. The expression of the weight function is generalised to include also a term penalising pixels with a high Euclidean distance value:

$$
\widehat{f}(g, d)=\alpha \exp \left(\beta_{1}(255-g)+\beta_{2} d\right)+\gamma,
$$

with $\alpha, \beta_{1}, \beta_{2}, \gamma \in \mathbb{R}$ and $d$ the average of the Euclidean distance values computed on the two incident pixels. The rationale supporting this decision is that the contour points will, with high probability, be inside region $\mathcal{R}$ and the probability of belonging to the breast contour decays with the increase of the Euclidean distance. The parameters $\alpha, \beta_{1}, \beta_{2}$ and $\gamma$ where experimentally tuned with the same method as before, yielding $\alpha=0.27, \beta_{1}=0.020, \beta_{2}=0.07$ and $\gamma=2$.

Non-parametric Unimodal Model. Intuitively, the breast contour is monotonically decreasing from one endpoint down to a minimum point, located approximately in the middle (of the breast) position, and from there it monotonically increases up to the other endpoint.

This simple perception about the shape of the breast can be enforced by running the shortest path algorithm in a phased process, instead of directly finding the path between the two given endpoints. To favour a first descending path, we find the shortest path between the external endpoint and a point located at the same column as the internal endpoint, but YGap rows below. We set yGap to twice the $x$-difference between the two endpoints (and therefore relatively robust to the size of the patient in the image). In the second step of the process, we compute a second shortest path between the point of the first path at the $x$-middle position and the internal endpoint. Fig. 2 illustrates the technique.

Note that, in spite of the name, the unimodal model does capture any fluctuation or irregularity in the evolution of contour, as it retains the flexibility of the shortest path process. Secondly, although the auxiliary point is positioned only twice width of the breast bellow the endpoint, the contour can, naturally, go below that point- - the actual shape is again captured by the shortest path. Finally, observe that the unimodal model relies only in Equation (4) to construct the weighted graph.

Non-parametric Template Matching. We now introduce a third non-parametric process. The template matching strategy tries to find the contour in the training set that best fits in the image. So, instead of constructing a region with all training contours, a single contour is selected as the prior. The steps of getting the most similar contour encompass creating an edge map from the image under evaluation and applying the distance transform on the extracted edge map, obtaining the Euclidean distance map $\Phi\left(x_{i}, y_{i}\right)$. The contour selected is the one minimizing the overall distance (normalized by its length):

$$
\min _{\mathcal{C}_{j}} \sum_{i \mid\left(x_{i}, y_{i}\right) \in \mathcal{C}_{j}} \Phi\left(x_{i}, y_{i}\right) / \text { length }\left(\mathcal{C}_{j}\right)
$$

The underlying assumption is that the training set is representative of the population of possible breast contours and by that a 'sufficiently' similar to the contour to be detected is present on the training set. The template matching procedure proceeds now 


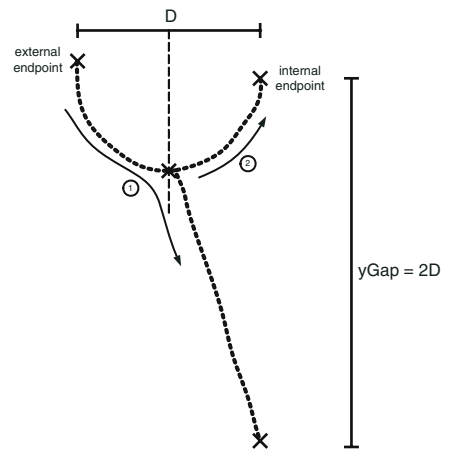

Fig. 2. Steps of the unimodal technique when applied on the left breast

exactly as the mask prior model, with the Euclidean distance computed in reference to the selected template contour. The template model relies on Equation 5 to construct the weight graph where the parameters $\alpha, \beta_{1}, \beta_{2}$ and $\gamma$ where experimentally tuned, yielding $\alpha=0.17, \beta_{1}=0.0208, \beta_{2}=0.055$ and $\gamma=2$.

\section{Automatic Detection of Endpoints}

The challenge now is to automatically extract the endpoints. In order to address this key problem we will assume, very reasonably, that a photo contains only the torso of the patient.

The position of the external endpoint of the breast contour can be assumed at the point of the body where the arm contour intersects the trunk contour. However, because patients are in the arms-down position, the arm's contour is almost indistinguishable from the trunk's contour. Therefore, we define the external endpoint of the breast contour as the highest point of the trunk contour.

The approach just delineated requires first the detection of the trunk contour. That may be searched among the strongest lines of gradient with approximate vertical direction.

\subsection{Trunk Contour Detection}

A strong vertical path corresponds to a path between two rows of the image, (almost) always through pixels with strong gradient values. Strong vertical paths are best modelled as paths between two regions $\Omega_{1}$ and $\Omega_{2}$, i.e. two rows of the image.

One may assume the simplifying assumption that the vertical paths do not zigzag back and forth, up and down. Therefore, the search may be restricted among connected paths containing one, and only one, pixel in each row between the two end-rows. Formally, let I be an $N_{1} \times N_{2}$ image with $N_{1}$ columns and $N_{2}$ rows; define a vertical path to be

$$
\mathbf{s}=\{(x(y), y)\}_{y=\Omega_{1}}^{\Omega_{2}} \text {, s.t. } \forall y|x(y)-x(y-1)| \leq 1,
$$

where $x$ is a mapping $x:\left[\Omega_{1}, \cdots, \Omega_{2}\right] \rightarrow\left[1, \cdots, N_{1}\right]$. That is, a vertical path is an 8 -connected path of pixels in the image from $\Omega_{1}$ to $\Omega_{2}$, containing one, and only one, pixel in each row of the image. 
The optimal vertical path that minimizes this cost can be found using dynamic programming (instead of resorting to a more complex algorithm for the generic shortest path problem). The first step is to traverse the image from the first to the last row and compute the cumulative minimum cost $\mathrm{C}$ for all possible connected paths lines for each entry $(i, j)$ :

$$
C(i, j)=\min \left\{\begin{array}{c}
C(i-1, j-1)+w\left(p_{i-1, j-1} ; p_{i, j}\right) \\
C(i, j-1)+w\left(p_{i, j-1} ; p_{i, j}\right) \\
C(i+1, j-1)+w\left(p_{i+1, j-1} ; p_{i, j}\right)
\end{array},\right.
$$

where $w\left(p_{i, j} ; p_{l, m}\right)$ represents the weight of the arc incident with pixels at positions $(i, j)$ and $(l, m)$. At the end of this process,

$$
\min _{i \in\left\{1, \cdots, N_{1}\right\}} C\left(i, N_{2}\right)
$$

indicates the end of the minimal connected path.

Stable Paths on a Graph. The procedure just delineated allows the computation of the single shortest path between two regions. However, we are interested in more than one strong path, possibly not exactly the shortest path (as the silhouette may constitute a shorter path). The concept of stable path allows the computation of a sort of local optimal paths.

Definition. A path $\mathcal{P}_{s, t}$ is a stable path between regions $\Omega_{1}$ and $\Omega_{2}$ if $\mathcal{P}_{s, t}$ is the shortest path between $s \in \Omega_{1}$ and the whole region $\Omega_{2}$, and $\mathcal{P}_{s, t}$ is the shortest path between $t \in \Omega_{2}$ and the whole region $\Omega_{1}$.

The naming of stable path has its roots in dynamical systems, as it resembles stable fixed points. If one considers the function $\mathcal{F}_{\Omega_{1} \rightarrow \Omega_{2}}\{\}$, mapping a node $s \in \Omega_{1}$ to a node $t \in \Omega_{2}$ by finding the shortest path $\mathcal{P}_{s, t}$ between $s$ and $\Omega_{2}$, with $t=\mathcal{F}_{\Omega_{1} \rightarrow \Omega_{2}}\{s\}$ as the end node of such shortest path, then

$$
\mathcal{G}_{\Omega_{1} \rightarrow \Omega_{1}}\{s\}=\mathcal{F}_{\Omega_{2} \rightarrow \Omega_{1}}\left\{\mathcal{F}_{\Omega_{1} \rightarrow \Omega_{2}}\{s\}\right\}=\mathcal{F}_{\Omega_{1} \rightarrow \Omega_{2}}\left\{\mathcal{F}_{\Omega_{2} \rightarrow \Omega_{1}}\{s\}\right\}=s
$$

if and only if $\mathcal{P}_{s, t}$ is a stable path. Note that the concept of stable path is valid for any graph and any two sub-graphs in general.

The computation of all stable paths in the graph derived from the image has only roughly twice the complexity of the shortest path computation. The first step corresponds verbatim to the computation of the shortest path presented at the beginning of this Section. In a second step one repeats the same procedure, traversing now the graph from the last to the first row. At the end of this process, if the two endpoints of a direct and reverse path coincide, we are in the presence of a stable point.

Phase 1 of the Endpoint Detection. We propose to apply the stable path concept to the bottom half of our photographs to find the trunk contour:

1. Compute the gradient of the image (see Fig. 3(a)).

2. Compute the shortest path between each point in the bottom row and the whole middle row of the gradient image (see Fig. 3(b)). 


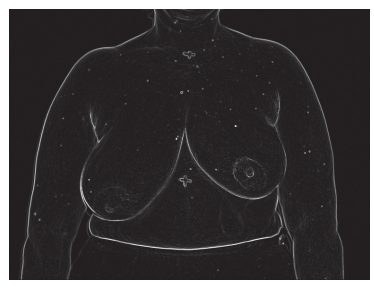

(a) Gradient

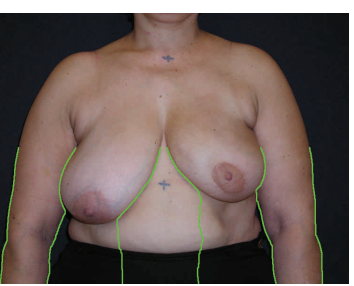

(d) Stable paths between the middle and bottom rows

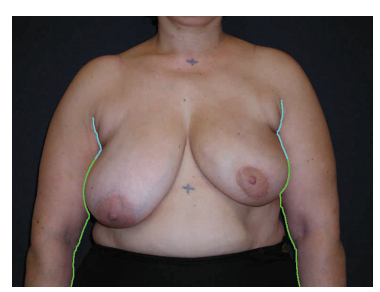

(g) The external endpoint of the breast contour is the highest point of the shortest path

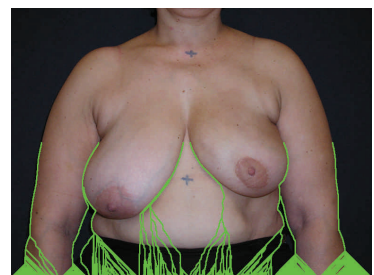

(b) Shortest paths from a pixel $t$ in the bottom line and the whole middle row $\Omega_{1}$, superimposed on the original image

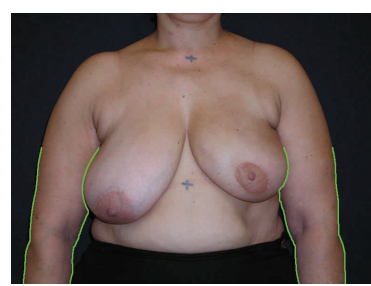

(e) Selected paths, superimposed on the original image

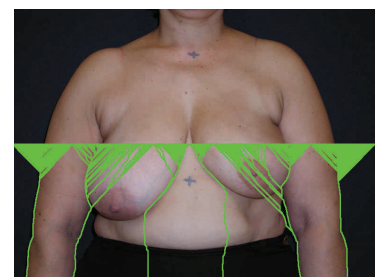

(c) Shortest paths from a pixel $s$ in the middle row and the whole bottom row $\Omega_{2}$, superimposed on the original image.

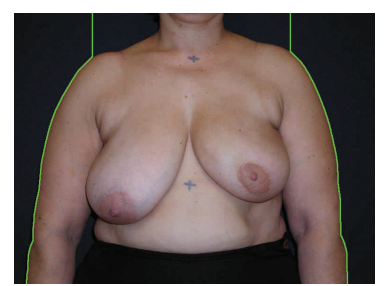

(f) Stable paths between the top and bottom rows

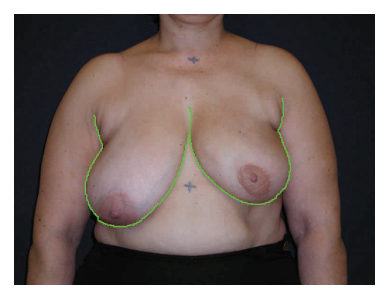

(h) Totally automated breast contour

Fig. 3. Results for a real photograph

3. Compute the shortest path between each point in the middle row and the whole bottom row of the gradient image (see Fig. 3(c)).

4. Discard all paths except for those common to steps 2 and 3 (see Fig. 3(d)). These first steps correspond to the computation of the stable paths between the middle and bottom rows.

5. Discard paths with a cost superior to half of the maximum possible cost (see Fig. 3(e)). Finally, the trunk contour is defined as the two contours closest to the middle of the photograph.

At the end of this phase we have already the position of the two trunk contours, but we have stopped the process at the middle of the image. It is important to stress that if the 
process was conducted between the bottom and top rows, the trunk contours would be lost, as the only strong paths between the top and bottom rows would be the external silhouette of the patient (see Fig. 3(f)) .

Phase 2 of the Endpoint Detection. To determine the top of the trunk contour, we need to continue the path produced in phase 1 until a certain condition is met. Towards that end, we propose to find the shortest path between the ending point of the stable contour found in phase 1 and row $R_{i}, R_{i}=$ middle_row, $\cdots$, top_row. We select the highest row for which the shortest path does not contain a long sequence (length_threshold) of consecutive pixels with low gradient (gradient_threshold). Fig. 3(g) illustrates the results obtained for the exemplificative photograph.

(length_threshold and gradient_threshold were set to 12 and 48, respectively).

Before applying the algorithm presented in Section 2 to compute the breast contour we need also the internal endpoint of the breast contour. This was estimated simply as the middle point between the two external endpoints. Finally, the computation of the breast contour yields the result presented in Fig. 3(h).

\section{Results}

The methodology proposed in this paper was assessed on a set of photographs from 120 patients. The photographs were collected in three different institutions in Portugal. All patients were treated with conservative breast surgery, with or without auxiliary surgery, and whole breast radiotherapy, with treatment completed at least one year before the onset of the study. Breast images were acquired employing a 4M pixel digital camera.

A mark was made on the skin at the suprasternal notch and at the midline $25 \mathrm{~cm}$ below the first mark (see Fig. 1). These two marks create a correspondence between pixels measured on the digital photograph and the length in centimetres on the patient. In order to investigate the possibility of defining an automated method of detecting the breast contour, a set of patients with known breast contour was required. Since, ideally, the automated method should correlate coherently with human assessment, eight different observers were asked to manually draw the contours. A software tool was developed specifically to assist on this job. The user defines the contour by positioning seventeen control points of cubic splines, see Fig. 4.

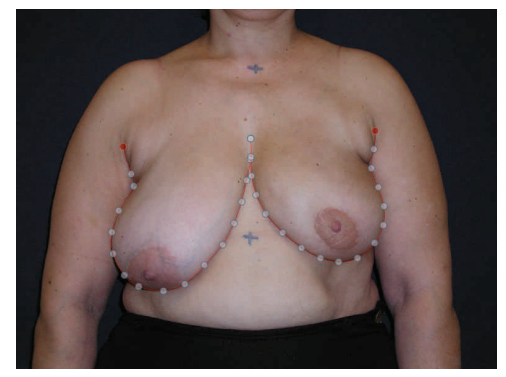

Fig. 4. Software for manual breast contour definition 


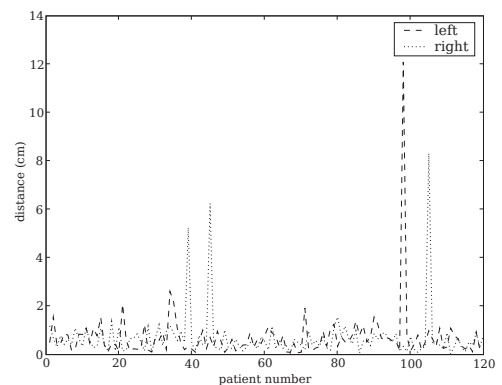

Fig. 5. Evolution of error $(\mathrm{cm})$ in the position of the external endpoints of the breast contour over 120 photographs

Before applying the proposed algorithm, each image was downsized to a constant width of 768 pixels, while keeping the aspect ratio. This improves the computational performance of the implementation of the software, without degrading the quality of the final result.

Fig. 5 shows the evolution of the error when estimating the external endpoints' position of the breast contour. The error in pixels was scaled to centimetres with the help of the marks made on the skin of the patient.

Table 1 summarizes the results.

Table 1. Mean, standard deviation and maximum value of errors in the position of the endpoints

\begin{tabular}{|c||c|c|c|}
\hline Error $(\mathrm{cm})$ & mean & std dev & max \\
\hline left endpoint & 0.7 & 1.1 & 12.1 \\
\hline right endpoint & 0.7 & 1.0 & 8.3 \\
\hline \hline total & 0.7 & 1.1 & 12.1 \\
\hline
\end{tabular}

It can be observed that the proposed algorithm has a very interesting performance. The average error is quite low, less than 1 centimetre. Fig. 6 shows some of the photographs for each the algorithm worked satisfactorily. It represents the result after the two phases of the algorithm. The highest point of the trunk contour provides the detected external endpoint of the breast contour. It is visible in patient \#35 that the algorithm is robust against cluttered background. It is also visible in Fig. 6 that, although the stable paths detected in phase 1 do not always correspond exactly to the trunk contour, the algorithm is still able to successfully detect the endpoints.

Nevertheless, four endpoints were clearly misplaced. These results, displayed in Fig. 7, bring to light some of the limitations of the current state of the proposed approach. In patients \#39, \#45 and \#105 the shortest path followed a 'wrong' contour, misplacing the endpoint. With patient \#98 the long hair created a false 'path' till the top of the image.

In a last set of experiments, the quality of the breast contour tracking algorithm was assessed. Instead of a simple subjective evaluation as provided in [5], we conducted a complete objective evaluation, based on the hausdorff and the average distances to 


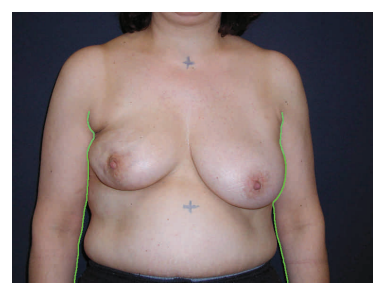

(a) Patient \#05

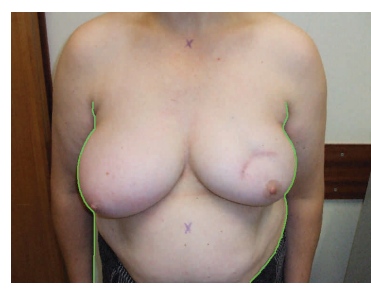

(b) Patient \#22

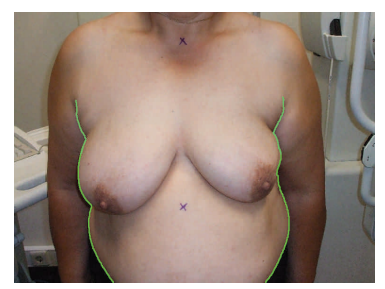

(c) Patient \#35

Fig. 6. Selected successful results

compare two contours. The hausdorff distance is defined as the "maximum distance of a set to the nearest point in the other set". Roughly speaking, it captures the maximum separation between the manual and the automatic contours.

Comparing the different models to detect the breast contour one can conclude from Table 2 that the simplest model, the unimodal prior models, and although its simplicity, exhibits a very competitive performance. As such, the experimental analysis is continued only with this model.

As observed in Fig. 8(a) and Table 2 the experimental values obtained for the hausdorff distance correlate well with the error on the endpoints. This fact means that, most of the times, the major error on the automatic contour is located on the endpoint, with

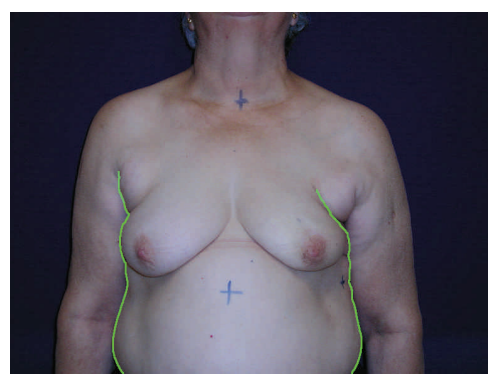

(a) Patient \#39

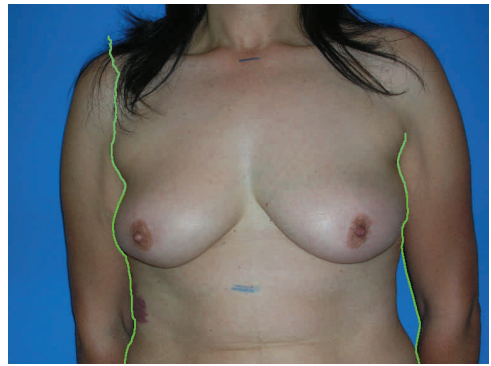

(c) Patient \#98

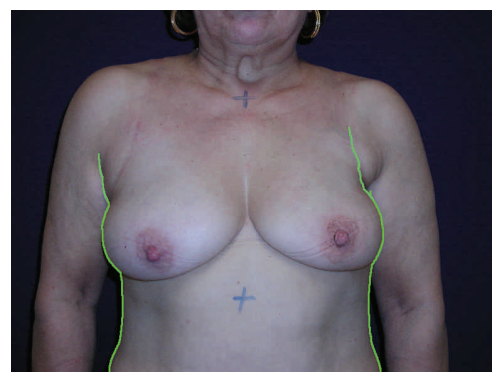

(b) Patient \#45

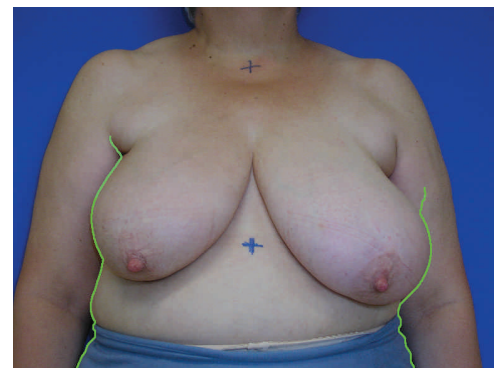

(d) Patient \#105

Fig. 7. All poor results 
Table 2. Performance in the position of the breast contour

\begin{tabular}{|c|c|c|c|c|c|c|c|}
\hline \multirow{3}{*}{$\begin{array}{l}\text { Method } \\
\text { Mask }\end{array}$} & & \multicolumn{3}{|c|}{$\begin{array}{l}\text { Average Error }(\mathrm{cm}) \\
\text { mean std. dev. max. }\end{array}$} & \multicolumn{3}{|c|}{$\begin{array}{l}\text { Hausdorff Error }(\mathrm{cm}) \\
\text { mean std. dev. max. }\end{array}$} \\
\hline & left breast & 0.25 & 0.61 & 5.52 & 1.81 & 1.78 & $\overline{14.04}$ \\
\hline & right breast & 0.27 & 0.68 & 4.91 & 1.78 & 1.87 & 13.22 \\
\hline \multirow{2}{*}{ Template } & left breast & 0.75 & 2.08 & 15.87 & 3.30 & 4.63 & 26.90 \\
\hline & right breast & 0.75 & 1.77 & 10.24 & 3.19 & 4.14 & 21.44 \\
\hline \multirow{2}{*}{ Unimodal } & left breast & 0.24 & 0.61 & 6.23 & 1.90 & 2.05 & 17.82 \\
\hline & right breast & 0.30 & 0.88 & 8.81 & 1.98 & 2.45 & 21.44 \\
\hline
\end{tabular}

the shortest path algorithm recovering the true contour rapidly. A clear exception is patient \#73, for which the error in the endpoints is negligible but the hausdorff distance between contours is very high. Here, the contour tracking algorithm missed to follow the contour, although it received proper endpoints.

The average distance between two contours captures better the perceived quality of the automatic breast contour. Here, the distance is averaged over the whole contour. Fig. 8(b) and Table 2 summarize these results.

The application of the breast contour detection after the automatic positioning of the ending points can result in different scenarios:

1. The endpoints are successfully located and the breast contour is correctly found. This desirable result is illustrated in Fig. 9(a).

2. The endpoints are successfully located but the algorithm misses to follow adequately the breast contour (see Fig. 9(b)).

3. The endpoints are poorly located but the algorithm rapidly finds and tracks the right breast contour (see Fig. 9(c)).

4. The endpoints are poorly located and the breast contour is incorrectly tracked (this scenario did not occur in the experimental set of photographs).

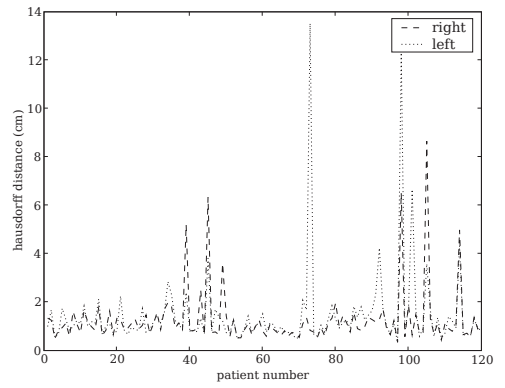

(a) Evolution of hausdorff distance $(\mathrm{cm})$ in the position of the breast contour over 120 photographs

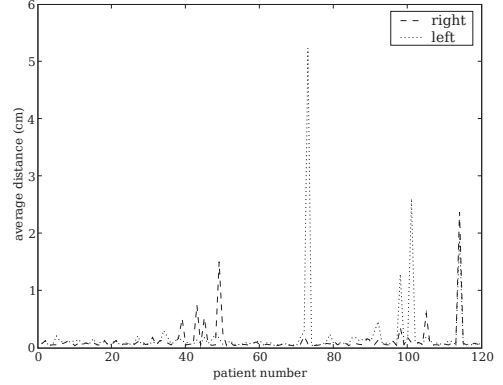

(b) Evolution of the average distance $(\mathrm{cm})$ in the position of the breast contour over 120 photographs

Fig. 8. Breast contour results 


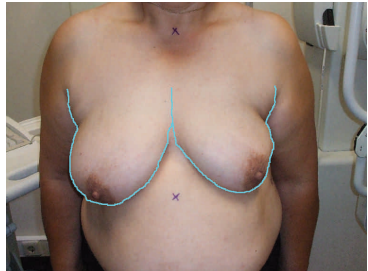

(a) Patient \#35

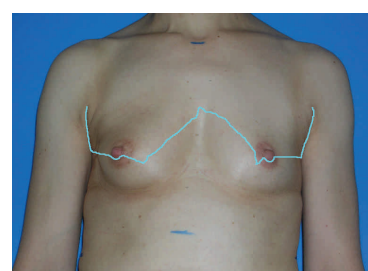

(b) Patient \#114

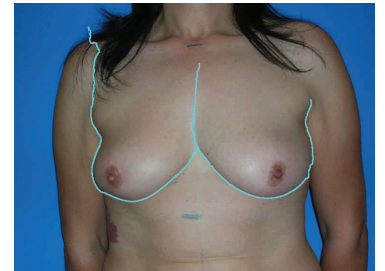

(c) Patient \#98

Fig. 9. Breast contour results

As expected by visual inspection patient of \#98 in Fig. 9(c), and although the error on the endpoint's location was high, the breast contour algorithm recovered rapidly the correct contour, translating into a final small average distance (but a high hausdorff distance).

The consequences of the diverse errors to the computer-aided medical system are different and have to be further studied. For example, one of the features used for the objective aesthetic evaluation of the BCCT is the difference between the levels of inferior breast contour points. This measure is quite robust over strong errors on the endpoints' position, as long as the breast contour is correctly tracked. Other features, such as the difference in the area of the breast are much more sensible to the positioning of the endpoints. One line of future investigation is the selection of features robust to the common errors of the automatic detection of notable points (contour endpoints, breast contour, nipples, etc) but still capturing adequately the aesthetic result, leading to a good classification performance.

\section{Conclusions}

A method has been described for applying graph concepts to the task of automatically extracting the breast contour in digital photographs of the torso of a patient, after being submitted to a breast cancer conservative treatment. In the proposed framework the problem of finding the endpoints of the breast contour is formulated as a problem of finding strong contours between two regions, a concept introduced here for the first time. The breast contour is found as the solution to the shortest problem on a graph, after conveniently modelling the image as a weighted graph. Preliminary results indicate a good performance in the tasks of finding the external endpoints of the contour and on detecting the breast contour. Future work will focus on improvements to the algorithm including generalizing the solution to other typical patient positions in these studies.

Acknowledgements. This work was partially funded by Fundação para a Ciência e a Tecnologia (FCT) - Portugal through project PTDC/EIA/64914/2006. 


\section{References}

1. Limbergen, E.V., Schueren, E.V., Tongelen, K.V.: Cosmetic evaluation of breast conserving treatment for mammary cancer. 1 . proposal of a quantitative scoring system. Radiotherapy and oncology 16, 159-167 (1989)

2. Christie, D.R.H., O’Brien, M.Y., Christie, J.A., Kron, T., Ferguson, S.A., Hamilton, C.S., Denham, J.W.: A comparison of methods of cosmetic assessment in breast conservation treatment. Breast 5, 358-367 (1996)

3. Al-Ghazal, S.K., Blamey, R.W., Stewart, J., Morgan, A.L.: The cosmetic outcome in early breast cancer treated with breast conservation. European journal of surgical oncology 25, 566-570 (1999)

4. Cardoso, J.S., Cardoso, M.J.: Towards an intelligent medical system for the aesthetic evaluation of breast cancer conservative treatment. Artificial Intelligence in Medicine 40, 115-126 (2007)

5. Cardoso, J.S., Cardoso, M.J.: Breast contour detection for the aesthetic evaluation of breast cancer conservative treatment. In: International Conference on Computer Recognition Systems (CORES 2007) (2007)

6. Sousa, R., Cardoso, J.S., da Costa, J.F.P., Cardoso, M.J.: Breast contour detection with shape priors. In: International Conference on Image Processing (ICIP 2008) (2008)

7. Dijkstra, E.W.: A note on two problems in connexion with graphs. Numerische Mathematik 1, 269-271 (1959)

8. Tsai, A., Yezzi, A., Wells, W., Tempany, C., Tucker, D., Fan, A., Grimson, W.E., Willsky, A.: A shape-based approach to the segmentation. of medical imagery using level sets. IEEE Transactions on Medical Imaging 22, 137-154 (2003)

9. Dydenko, I., Jamal, F., Bernard, O., D’Hooge, J., Magnin, I.E., Friboulet, D.: A level set framework with a shape and motion prior for segmentation and region tracking in echocardiography. Medical Image Analysis 10, 162-177 (2006)

10. Yan, P., Kassim, A.A.: Medical image segmentation using minimal path deformable models with implicit shape priors. IEEE Transactions on Information Technology in Biomedicine 10, 677-684 (2006) 